\title{
Adrenal Myelolipoma Associated with Spherocytosis: An Extremely Rare Case
}

\author{
Youness Chakir* ${ }^{(0)}$, El Mahdi Graiouid, Jandou Issam, Mohamed Dakir, Adil Debbagh, \\ Rachid Aboutaieb
}

Department of Urology, UHC Ibn Rochd, Casablanca, Morocco

Email: *dr.chakir.youness@gmail.com

How to cite this paper: Chakir, Y., Graiouid, E.M., Issam, J., Dakir, M., Debbagh, A. and Aboutaieb, R. (2019) Adrenal Myelolipoma Associated with Spherocytosis: An Extremely Rare Case. Case Reports in Clinical Medicine, 8, 281-284.

https://doi.org/10.4236/crcm.2019.811034

Received: October 20, 2019

Accepted: November 5, 2019

Published: November 8, 2019

Copyright $\odot 2019$ by author(s) and Scientific Research Publishing Inc. This work is licensed under the Creative Commons Attribution International License (CC BY 4.0). http://creativecommons.org/licenses/by/4.0/

\begin{abstract}
Adrenal myelolipoma is a benign, non-secreting tumor composed of adipose and myeloid tissue that can be complicated by retroperitoneal pain or hemorrhage. Its diagnosis is carried by the computed tomography (CT) and confirmed histologically. Surgery may be necessary in case of large volume, symptomatic mass or complication. We report the case of a 36-year-old patient who has a combination of myelolipoma and hereditary spherocytosis, which is extremely rare in the world literature. Computed tomography guided the diagnosis and surgical excision was performed because of the volume of the mass and its symptomatic nature. The evolution at two years was without recurrences.
\end{abstract}

\section{Keywords}

Non-Secreting Tumor, Adipose Tissue, Myeloid Tissue, Adrenal Myelolipoma

\section{Introduction}

Adrenal myelolipoma is a rare tumor, its first description in the literature dates from 1905, its composition is made of adipose and hematopoietic tissue [1]. Its diagnosis is based on a CT scan that identifies the fat percentage. However, these radiological aspects may lead to diagnostic confusion with kidney angiomyolipoma, lipoma, and liposarcoma, it is often unexpectedly detected [2]. Hereditary spherocytosis (HS) is characterized by increased osmotic fragility and enhanced membrane loss of red blood cells (RBC) due to defective membrane protein complexes. The majority are small and asymptomatic, giant cases are described, the association with spherocytosis is rare.

We report a new case of giant adrenal myelolipoma associated with spherocy- 
tosis and discuss the diagnostic and therapeutic aspects of this rare condition.

\section{Observation}

Mr. M.A, 36 years old followed for hereditary spherocytosis, consulted for low back pain and the awkward feeling of awkward gravity during walking. On examination, the patient was in good general condition with right lumbar, icteric contact and splenomegaly were observed. The abdominal ultrasound revealed a hyperechoic mass of $11 \times 10 \times 3 \mathrm{~cm}$ at the expense of the right adrenal lodge pushing the kidney down and in and the liver up. Computed tomography showed a mixed heterogeneous density right mass between the liver and the right kidney, with a weakly heterogeneous contrast medium measuring $13 \times 8 \times$ 4 (Figure 1). The hydro electrolytic balance, as well as the hormonal assays, was normal. Given these radiological criteria, the diagnosis of adrenal myelolipoma is suspected. Surgical excision of the mass was performed because of its volume and symptomatic nature. It weighed $606 \mathrm{~g}$ and measured $13 \times 9 \times 3 \mathrm{~cm}$. It was nodular with a hemorrhagic, soft, greasy appearance. On histological examination, the formation is limited by a fibrous capsule. The long-term follow-up was simple and follow-up of the patient did not reveal recurrence after two years of evolution.

\section{Discussion}

Adrenal myelolipoma is a rare benign tumor first described by Gierke [1]. Its pathophysiology is thought to be metaplasia of adrenal cortical cells into reticuloendothelial cells in response to infection, chronic stress or adrenal degeneration [2]. In most cases, the tumor is fortuitously discovered during an imaging

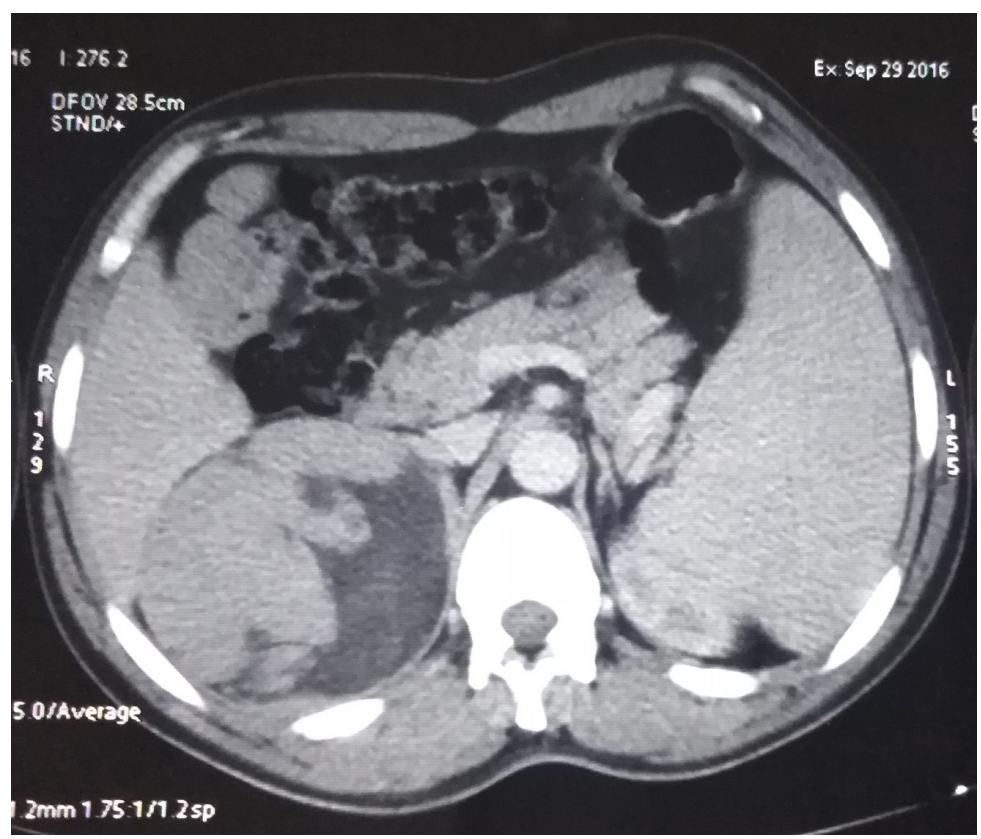

Figure 1. Abdominal CT showing myelolipoma and splenomegaly. 
test for another indication. Symptoms such as flank pain, heaviness, hematuria, or palpable mass result from tumor compression, necrosis, or spontaneous retroperitoneal hematoma [3], the size varies from one centimeter to more than thirty centimeters but the majority of the masses are less than five centimeters in size [4] [5]. Rarely isolated, adrenal myelolipoma is often associated with various conditions: hypertension, obesity, various endocrinopathies such as Cushing disease, Conn's syndrome or pheochromocytoma and non-endocrine disorders [6] [7]. Generally, adrenal myelolipomas are not concomitant with hematologic disease [5]. However, in the present case, a giant adrenal myelolipoma was associated with hereditary spherocytosis. The association with spherocytosis is rarely described in the literature [8].

Although the adrenal myelolipoma in the present case may have been incidentally associated with hereditary spherocytosis, the hematopoietic stimulus due to severe hemolysis in spherocytosis might induce extramedullary hematopoiesis in the adrenal gland. In the literature three etiologic theories of adrenal myelolipoma have been presented: 1) degeneration of differentiated cortical cells; 2) metaplasia of mesenchymal cortical cells; 3) choristoma theory: misplacement of hematopoietic cells [8]. We believe that our case supports the choristoma theory as an etiology of adrenal myelolipoma.

The radiological diagnosis of adrenal myelolipoma relies on the identification of the fatty portion of the tumor on computed tomography, but these aspects are not pathognomonic. Magnetic resonance imaging (MRI) allows a characterization of the various components of the myelolipoma by finding a high signal in $\mathrm{T} 1$ and signal attenuation in T2 for the fat component as well as a low signal in $\mathrm{T} 1$ and an intermediate signal on $\mathrm{T} 2$-weighted sequences for the hematopoietic component [9] [10].

The recommendations suggest surgery for tumors larger than $7 \mathrm{~cm}$, because of the risk of spontaneous rupture or intraperitoneal hemorrhage, tumors less than $4 \mathrm{~cm}$ must be followed by an annual abdominal CT scan [11] [12]. In our case, mass surgery was performed because of the symptomatology and its volume.

\section{Conclusion}

Myelolipoma is a rare tumor, the association with hereditary spherocytosis is poorly described in the literature. Our case was treated by surgical excision with uncomplicated follow-up at two years of follow up. The hematopoietic stimulus of the hereditary spherocytosis might have been associated with the development of adrenal myelolipoma in the present case.

\section{Authors' Contributions}

Youness Chakir wrote the article, El Mahdi Graiouid contributed to the taking of the photos, Dakir Mohamed, Adil Debbagh and Rachid Aboutaieb contributed to the therapeutic management of the patient and the writing of this document. All authors have read and approved the final version of this manuscript. 


\section{Statement}

The patient had given his consent for the Case report to be published.

\section{Conflicts of Interest}

The authors declare no competing interest.

\section{References}

[1] Gierke, E. (1905) Uber Knochenmarksgwebe in der Nebenniere. Beitrage zur pathologischen anatomie, 7, 311.

[2] Rafiq, B., et al. (2017) Adrenal Myelolipoma: About a Case. The Pan African Medical Journal, 28, 153. https://doi.org/10.11604/pamj.2017.28.169.13033

[3] Wrightson, W.R., Hahm, T.X., Hutchinson, J.R. and Chedle, W. (2002) Bilateral Giant Adrenal Myelolipomas: A Case Report. The American Surgeon, 68, 588-589.

[4] Enzinger, F.M. and Sharen, W.W. (1995) Benign Lipomatous Tumors. In: Enzinger, F.M. and Sharen, W.W., Eds., Soft Tissue Tumors, Mosby, St. Louis, 3rd Edition, 409-410.

[5] Meaglia, J.P. and Schmidt, J.D. (1992) Natural History of an Adrenal Myelolipoma. The Journal of Urology, 147, 1089-1090. https://doi.org/10.1016/S0022-5347(17)37482-7

[6] El Mezni, F., Zermani, R., Touati, A., Zakraoui, A., Chebil, M., Dziri, C. and Ayed, M. (1995) Adrenal Myelolipoma: About 3 Cases. The Journal of Urology (Paris), 101, 203-206.

[7] Han, M., Burnetta, L., Fishman, E.K. and Marshallf, F. (1995) The Natural History and Treatment of Adrenal Myelolipoma. The Journal of Urology, 153, 129-132.

[8] Dieckmann, K.-P., Hamm, B., Pickartz, H., Jonas, D. and Bauer, H.W. (1987) Adrenal Myelolipoma: Clinical, Radiologic, and Histologic Features. Urology, 29, 1-8. https://doi.org/10.1016/0090-4295(87)90587-5

[9] Sehili, S., Jemli, C.H., Jouini, S., Shammakhi, N., Bouzaidi, K. and Daghfous, M.H. (2001) Adrenal Myelolipoma: About 4 Cases. Magazine Medicale, 21, 9-11.

[10] Zidi, B., Bouziani, A., Louzir, B., Ben Hamadi, F., Machghoul, S. and Bahri, M. (1995) Adrenal Incidentalomas: About 4 Personal Observations. Medical Tunisia, 73, Article ID: 391402.

[11] Kenney, P.J., Wagner, B.J., Rao, P. and Heffess, C.S. (1998) Myelolipoma: CT and Pathologic Features. Radiology, 208, 87-95. https://doi.org/10.1148/radiology.208.1.9646797

[12] Nabi, J., Rafiq, D., Authoy, F.N. and Sofi, G.N. (2013) Incidental Detection of Adrenal Myelolipoma: A Case Report and Review of Literature. Case Reports in Urology, 2013, Article ID: 789481. https://doi.org/10.1155/2013/789481 